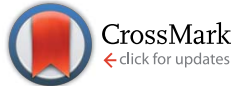

Cite this: Chem. Sci., 2015, 6, 2211

Received 8th January 2015

Accepted 5th February 2015

DOI: $10.1039 / \mathrm{c} 5 \mathrm{sc} 00071 \mathrm{~h}$

www.rsc.org/chemicalscience

\section{Redefining q: quaternary ammonium cross sectional area (XSA) as a general descriptor for transport-limiting PTC rate approximations $\uparrow$}

\begin{abstract}
S. E. Denmark* and J. J. Henle
The relationships of several key molecular descriptors with the rate of the O'Donnell alkylation under phase transfer catalysis (PTC) have been investigated. The most common parameter used to predict PTC rates, the ammonium ion accessibility, $\boldsymbol{q}$, is defined in such a way that limits its use to straight-chain tetraalkylammonium catalysts. To find a general descriptor of rate, eight linear, symmetrical tetraalkylammonium cations were examined to determine if a model containing broadly applicable descriptors could be found. The catalytic activity of these salts was determined under PTC conditions (operating under an interfacial, transport-rate limiting mechanism) and was compared with these molecular descriptors. Models could be generated from the ammonium ion accessibility parameter $q$ and the amphiphilic cross sectional area descriptor (XSA), and each gave a correlative model predicting the rate of alkylation. However, a similar model cannot be generated from a descriptor that is a direct measure of ammonium ion accessibility, the solvent accessible ammonium surface area (NC4_SA). These models lead to the conclusion that $q$ must approximate catalyst properties other than ammonium ion accessibility. Additionally, the relationship between XSA and rate demonstrates that XSA approximates the complex behavior of ammonium ions at the interfacial region of a biphasic system, allowing for its use as a general descriptor for transport-limiting PTC rate approximations.
\end{abstract}

\section{Introduction}

Phase-transfer catalysis (PTC) is an extremely useful method for performing nearly any type of reaction involving an ionic starting material or intermediate with a neutral partner. ${ }^{1}$ In PTC, two immiscible phases separate reactive reagents. Reaction and catalysis is enabled by a phase-transfer reagent; typically a quaternary ammonium or phosphonium salt that facilitates the transfer of a reactive agent between the two immiscible phases. Several characteristics of PTC are attractive for industrial applications including ease of scalability, operational simplicity, and ease of separation of products from byproducts. A useful aspect of PTC is the ability to catalyze a wide variety of reaction types, ranging from redox processes to $\mathrm{C}-\mathrm{C}$ bond forming reactions. ${ }^{2}$ An important type of $\mathrm{C}-\mathrm{C}$ bond forming reaction is the alkylation of carbanions formed under PTC with inorganic hydroxide bases, termed "hydroxide-initiated PTC". ${ }^{3}$ Employing hydroxide bases to generate anions that are typically formed using strong bases (LDA, NaH, etc.) highlights the advantages in operation and scalability using PTC.

Roger Adams Laboratory, Department of Chemistry, University of Illinois, Urbana, Illinois, 61801, USA. E-mail: sdenmark@illinois.edu

$\dagger$ Electronic supplementary information (ESI) available. See DOI: $10.1039 / \mathrm{c} 5 \mathrm{sc} 00071 \mathrm{~h}$
Hydroxide-initiated PTC has been highly successful in the catalytic, enantioselective alkylation of enolates. ${ }^{4}$

The versatility of PTC of reactions involving ionic intermediates places it at the forefront of general utility within organic synthesis. However, a fundamental understanding of the structural features that lead to catalytic activity and selectivity is still lacking. This deficiency arises from the inherent complexity of the roles of catalyst, base, and substrate in a biphasic mixture. For PTC to be successful, both physical (transport) and chemical (reactivity) aspects must be considered. In hydroxideinitiated PTC, two limiting mechanisms have been proffered to explain the activity of the catalyst: the extraction mechanism proposed by Starks ${ }^{5}$ and the interfacial mechanism proposed by Makosza $^{6}$ (Fig. 1). The extraction mechanism postulates that quaternary ammonium species $\left(\mathbf{Q}^{+}\right)$acts to transfer hydroxide $\left(\mathbf{O H}^{-}\right)$to the organic phase to generate the ammonium enolate $\left(\mathbf{Q}^{+} \mathbf{E n}^{-}\right)$(Fig. 1, left). In the interfacial mechanism, the enolate is generated at the interfacial region between the organic and aqueous phases by reaction with hydroxide (Fig. 1, right). The quaternary ammonium salt then effects ion exchange and transfers the enolate from the interfacial region (desorption) into the organic phase, where alkylation can take place. Numerous kinetic studies have provided evidence for both of these mechanisms, depending on reaction conditions. ${ }^{7}$ For practical purposes, a general guideline is that PTC reactions of 


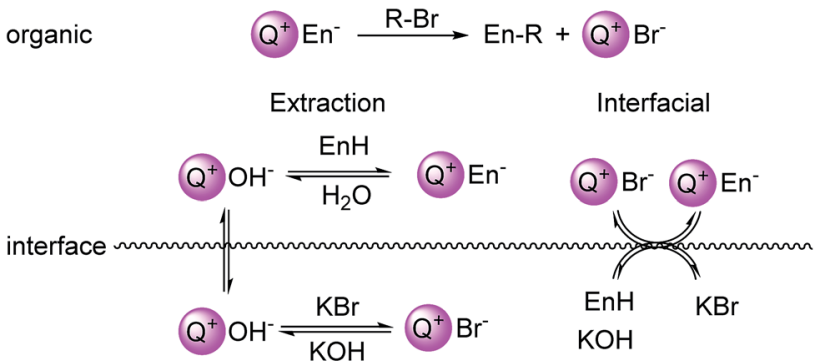

aqueous

Fig. 1 Left: depiction of the Stark's extraction mechanism for hydroxide-initiated PTC. Right: depiction of Makosza's interfacial mechanism for hydroxide-initiated PTC. En = enolate.

carbon acids with a $\mathrm{p} K_{\mathrm{a}}$ range $18<x<25$ undergo reaction in the interfacial mode.

Despite extensive kinetic investigation, a predictor of catalyst activity in hydroxide-initiated PTC remains elusive as no general design parameters that lead to high catalytic activity have been identified. For PT-catalysts, structure-activity relationships (SAR) have been identified for straight-chain tetraalkylammonium $\left(\mathrm{R}_{4} \mathrm{~N}^{+}\right)$salts that promote reaction of small hydrophilic nucleophiles. However, such studies involving hydroxide-initiated PTC are primitive by comparison. ${ }^{1 e}$ The most commonly invoked structural features of $\mathrm{R}_{4} \mathrm{~N}^{+}$salts in SAR studies is the ammonium ion accessibility parameter $\boldsymbol{q}{ }^{8}$ The parameter $\boldsymbol{q}$ is a structural descriptor of straight-chain $\mathrm{R}_{4} \mathrm{~N}^{+}$species originally introduced by Rabinovitz and Halpern in a series of studies that aimed to define the limits of the spectrum between the two aforementioned mechanisms. ${ }^{9}$ The parameter $\boldsymbol{q}$ is defined as the sum of the reciprocal of the number of carbons in the $\mathrm{R}_{4} \mathrm{~N}^{+}$ chains (eqn (1), where $C_{n}$ is the number of carbon atoms in chain $n$ ). After surveying many hydroxide-initiated PTC reactions, it was observed that most active catalysts had $\boldsymbol{q}$-values between 1.5 and $2.0{ }^{10}$ Generally, a $\mathrm{R}_{4} \mathrm{~N}^{+}$ion is considered accessible if $\boldsymbol{q}>1$.

$$
\boldsymbol{q}=\frac{1}{C_{1}}+\frac{1}{C_{2}}+\frac{1}{C_{3}}+\frac{1}{C_{4}}
$$

Although $\boldsymbol{q}$ may exist as a general descriptor for straightchain $\mathrm{R}_{4} \mathrm{~N}^{+}$ion activity in hydroxide initiated PTC, the definition of the parameter prevents its use in the case of more complex quaternary ammonium species. As the logic behind the derivation of $\boldsymbol{q}$ was never clarified, extension of the parameter is difficult. To address this problem, previous work in these laboratories introduced a more general catalyst structure descriptor to correlate with rate data. ${ }^{\mathbf{1 1}}$ This type of descriptor is of paramount importance in employing asymmetric PTC (APTC) reactions that require chiral, branched, and substituted quaternary ammonium species. The ability to select catalysts that can out-compete a background reaction is fundamental to chiral catalyst design. Although many descriptors were evaluated as potential replacements for $\boldsymbol{q}$, it was found that $\boldsymbol{q}$ correlates well with the solvent accessible ammonium ion surface area descriptor (NC4_SA, Fig. 2). The NC4_SA descriptor

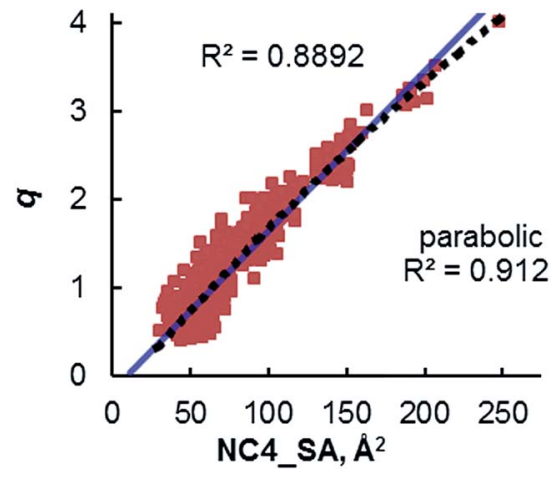

Fig. 2 Relationship of the NC4_SA values of all straight-chain alkyl $\mathrm{R}_{4} \mathrm{~N}^{+}$catalysts containing 4-40 carbon atoms and $q$. Adapted with permission from S. E. Denmark, N. D. Gould, L. M. Wolf, J. Org. Chem., $2011,76,4337-4357$

calculates the solvent accessible surface area (ASA) of the atoms that comprise the ammonium ion (nitrogen and neighboring carbon atoms), a direct measure of ammonium ion accessibility. ${ }^{12}$

Once the correlation was established, a kinetic study was performed to investigate the relationship of NC4_SA and other molecular descriptors with rate. This previous work ${ }^{\mathbf{1 1}}$ used the O'Donnell benzylation of glycine Schiff base $\mathbf{1}$ as a model reaction (Fig. 3) to study using these descriptors. ${ }^{13}$ Catalytic activity data was taken under conditions such that the reaction exhibited stir rate dependence with a highly reactive nucleophile ensuring that the reaction is transport-rate limiting (i.e. alkylation is not the rate-limiting step). ${ }^{11 c-e}$ In the original report, it was shown that NC4_SA had a moderate correlation with the rate of the O'Donnell alkylation in conjunction with
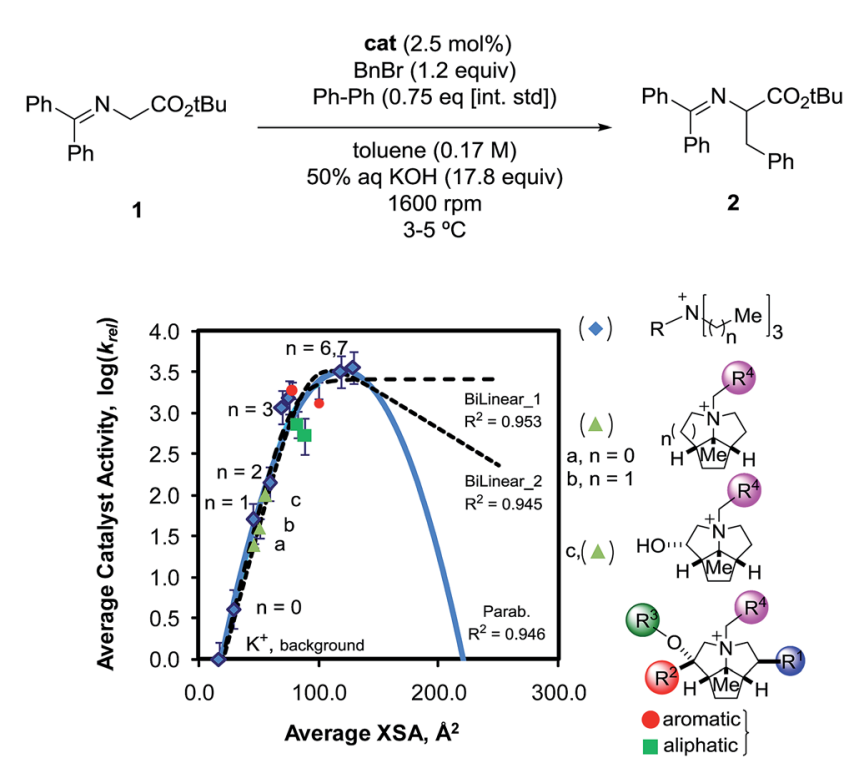

Fig. 3 Observed correlation between XSA descriptor and catalyst activity in the O'Donnell alkylation. Adapted with permission from S. E. Denmark, N. D. Gould, L. M. Wolf, J. Org. Chem., 2011, 76, 4337-4357. 
other molecular descriptors. ${ }^{\mathbf{1 1}}$ However, it was found in the original study that the amphiphilic cross-sectional area ${ }^{\mathbf{1 4}}$ descriptor (XSA) acted as a potential single descriptor for rate (Fig. 3) exceeding the correlation observed in rate models containing NC4_SA.

The XSA descriptor first determines the amphiphilic axis (the axis between the center of mass of hydrophobic and polar atoms, respectively), then a plane is defined at the center of this axis, lying orthogonal to the axis. The area of this plane circumscribing the van der Waals surface of the molecule is the XSA value in $\AA^{2}$ (Fig. 4). The XSA descriptor models behavior of amphiphilic molecules at the highly anisotropic interface between a polar and nonpolar environment, and is thus ideally suited to model catalysts operating in the interfacial PTC mechanism. $^{15}$

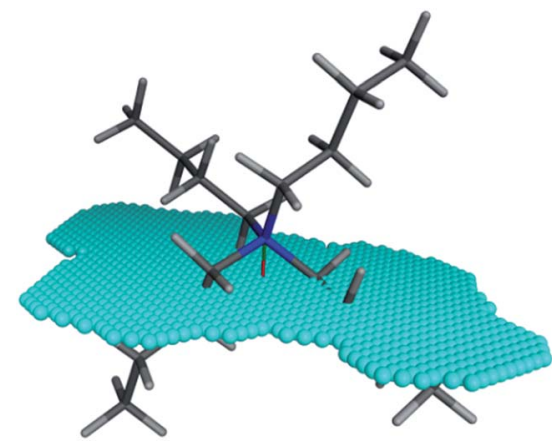

Fig. 4 Amphiphilic cross sectional area (XSA) of the tetrabutylammonium cation. The amphiphilic axis is shown as the red-green line, and the cross section plane is denoted by the blue spheres.

A linear increase of rate with increasing XSA was observed until $75 \AA^{2}$ was reached, and which point the reaction rate leveled off, with the larger $\mathrm{R}_{4} \mathrm{~N}^{+}$salts having a maximum area of $131 \AA^{2}$. The original work concluded that over the observed range, XSA is proportional to the rate at which the $\mathbf{Q}^{+} \mathbf{E n}^{-}$ species can undergo the desorption process from the interfacial region. It is proposed that the levelling of rate results from the rate of desorption approaching the diffusion limit. From this data, three nonlinear correlative models were found, shown in Fig. 3.

Depending on the model considered, different interpretations of what XSA physically represents are possible. If the constant rate trend was found to continue (Fig. 3, Bilinear_1), it would indicate that XSA is solely modeling desorption from the interface. If the rate began decreasing as XSA increases (fitting models similar to Bilinear_2 and Parab, Fig. 3), this would indicate that XSA models adsorption to and desorption from the interface. The goal of the study herein was to determine the kinetic behavior of PTC catalysts in a higher $\left(>130 \AA^{2}\right)$ XSA regime in the O'Donnell benzylation reaction and determine to which model, if any, the kinetic data conforms. Additionally, other descriptors such as $\boldsymbol{q}$ and NC4_SA were also investigated for correlative relationships with PTC rates.

\section{Results and discussion}

To further elucidate the physical interpretation of the XSA descriptor with respect to transport-limiting PTC, catalysts possessing larger XSA's would be evaluated for PTC activity. Quaternary ammonium catalysts were identified through a selective in silico quaternary ammonium ion screen. The library consisted of candidates derived from the common unsymmetrical, complex $\mathrm{R}_{4} \mathrm{~N}^{+}$cores (Cinchona alkaloids $(3,4)$, cyclopentapyrrolizidines (5), ${ }^{11}$ Maruoka catalysts (6), ${ }^{16}$ etc.) as well as commercially available straight-chain $\mathrm{R}_{4} \mathrm{~N}^{+}$salts (7) (Fig. 5).

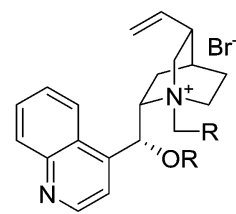

$80-120 \AA^{2}$

3<smiles>[R][N+]1([R])Cc2ccc3ccccc3c2-c2c(ccc3ccccc23)C1</smiles>

$70-150 \AA^{2}$

6

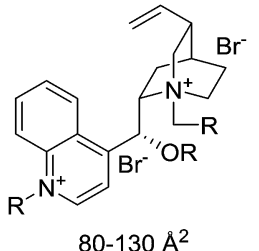

4

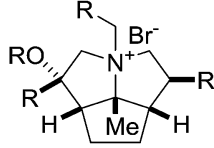

$80-120 \AA^{2}$

5
Fig. 5 The tetraalkylammonium salts from a variety of scaffold libraries used for in silico screening of various descriptors. The values given are the general ranges of XSA values for each scaffold. These values are calculated using the method described in the ESI. $\dagger$

None of the complex, polycyclic scaffolds yielded structures with calculated XSA's greater than $150 \AA^{2}$. However, several, large XSA salts were found in the straight-chain $\mathrm{R}_{4} \mathrm{~N}^{+}$library. In view of their commercial availability, tetradecylammonium bromide (TDAB), tetradodecylammonium bromide (TDoDAB), and tetrakis(hexadecyl)ammonium bromide (THexDAB) were chosen as the large XSA catalyst representatives (Table 1, entries 6-8). In addition, it was deemed prudent to reassess the activity of the straight-chain $\mathrm{R}_{4} \mathrm{~N}^{+}$catalysts previously reported to ensure that accurate correlations were obtained. The calculated average XSA values are reported in Table $1 .^{\mathbf{1 7}}$

The solvent accessible ammonium ion surface area (NC4_SA) was also calculated for these large $\mathrm{R}_{4} \mathrm{~N}^{+}$catalysts. The results of these calculations show that the ammonium surface area remains invariant for larger values of XSA (Table 1). Thus, as XSA increases with increasing chain length in the $\mathrm{R}_{4} \mathrm{~N}^{+}$species, a decrease in NC4_SA is observed, followed by a relatively constant region once XSA $\sim 75 \AA^{2}$ is reached (Fig. 6). This suggests that PT-catalysts with increasingly large XSA have similar ammonium ion accessibilities.

The selected straight-chain $\mathrm{R}_{4} \mathrm{~N}^{+}$salts were then employed as catalysts in the O'Donnell benzylation reaction. Each catalyst was tested in triplicate, with the resulting data having an 
Table 1 Amphiphilic cross sectional area (XSA) and solvent accessible ammonium ion surface area (NC4_SA) values for the straight-chain $\mathrm{R}_{4} \mathrm{~N}^{+}$salts

\begin{tabular}{lllll}
\hline Entry & Catalyst $\left(\mathrm{R}_{4} \mathrm{~N}^{+}\right)$ & Catalyst & XSA $^{a, b}\left(\AA^{2}\right)$ & $\mathrm{NC}_{-} \mathrm{SA}^{a, b}\left(\AA^{2}\right)$ \\
\hline 1 & $\left(\mathrm{CH}_{3}\right)_{4} \mathrm{~N}^{+}$ & TMAB & 29.00 & 247.24 \\
2 & $\left(\mathrm{C}_{2} \mathrm{H}_{5}\right)_{4} \mathrm{~N}^{+}$ & TEAB & 41.81 & 94.88 \\
3 & $\left(\mathrm{C}_{4} \mathrm{H}_{9}\right)_{4} \mathrm{~N}^{+}$ & TBAB & 72.22 & 52.23 \\
4 & $\left(\mathrm{C}_{6} \mathrm{H}_{13}\right)_{4} \mathrm{~N}^{+}$ & THexAB & 101.17 & 48.22 \\
5 & $\left(\mathrm{C}_{7} \mathrm{H}_{15}\right)_{4} \mathrm{~N}^{+}$ & THeptAB & 114.14 & 48.26 \\
6 & $\left(\mathrm{C}_{8} \mathrm{H}_{17}\right)_{4} \mathrm{~N}^{+}$ & TOAB & 128.83 & 47.68 \\
7 & $\left(\mathrm{C}_{10} \mathrm{H}_{21}\right)_{4} \mathrm{~N}^{+}$ & TDAB & 152.03 & 44.64 \\
8 & $\left(\mathrm{C}_{12} \mathrm{H}_{25}\right)_{4} \mathrm{~N}^{+}$ & TDoDAB & 174.34 & 39.10 \\
9 & $\left(\mathrm{C}_{16} \mathrm{H}_{33}\right)_{4} \mathrm{~N}^{+}$ & THDAB & 205.27 & 23.08
\end{tabular}

${ }^{a}$ Calculated using MOE 2013.08 using MMFF94x. ${ }^{b}$ Values are averages calculated from a conformer library containing conformers within 20 $\mathrm{kcal} \mathrm{mol}^{-1}$ from equilibrium conformer.

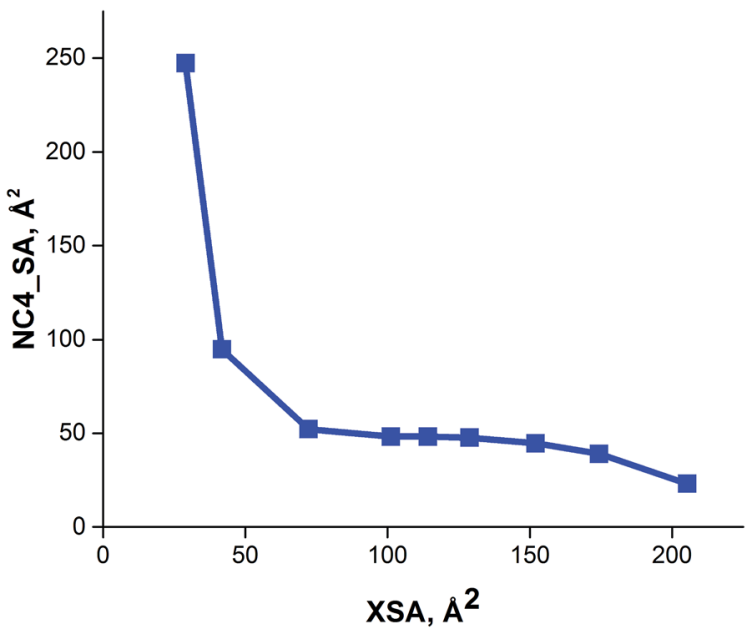

Fig. 6 Relationship between cross sectional area (XSA) and solvent accessible ammonium ion surface area (NC4_SA) descriptors.

average percent error of $3.19 \% .{ }^{18}$ As in the previous study, the metric of catalytic activity was the half-life $\left(t_{1 / 2}\right)$ of the reaction at a constant stirring rate. The kinetic data obtained for these catalysts is shown in Table 2 . Once again, the catalysts with the highest activity were THAB, THeptAB (heptyl) and TOAB (entries 3-5). After TOAB, an increase in the chain length of the $\mathrm{R}_{4} \mathrm{~N}^{+}$ catalysts resulted in decreased activity (entries 6-8).

The relationship between the rate data and XSA was examined. Clearly, the rate does not remain constant (after reaching the maximum observed rate) with increasing XSA, but instead, a nonlinear relationship is observed is observed between rate and XSA (Fig. 7). The best $\mathrm{t}^{19}$ model was observed with a third order polynomial (cubic function) with an $R^{2}$ of 0.988 (Fig. 7a). ${ }^{20} \mathrm{~A}$ parabolic model was generated, giving a similarly high $R^{2}$ of 0.950 (Fig. 7b). A bilinear model was investigated, but gave poorer observed correlation $\left(R^{2}=0.889\right)$ (Fig. 7c). While the parabolic model shows good correlation, the cubic model appears to more accurately describe the behavior of the most active catalysts as well as the least active. ${ }^{19}$ This is due to the
Table 2 Kinetic data for $\mathrm{R}_{4} \mathrm{~N}^{+}$PTC catalysts ${ }^{a}$

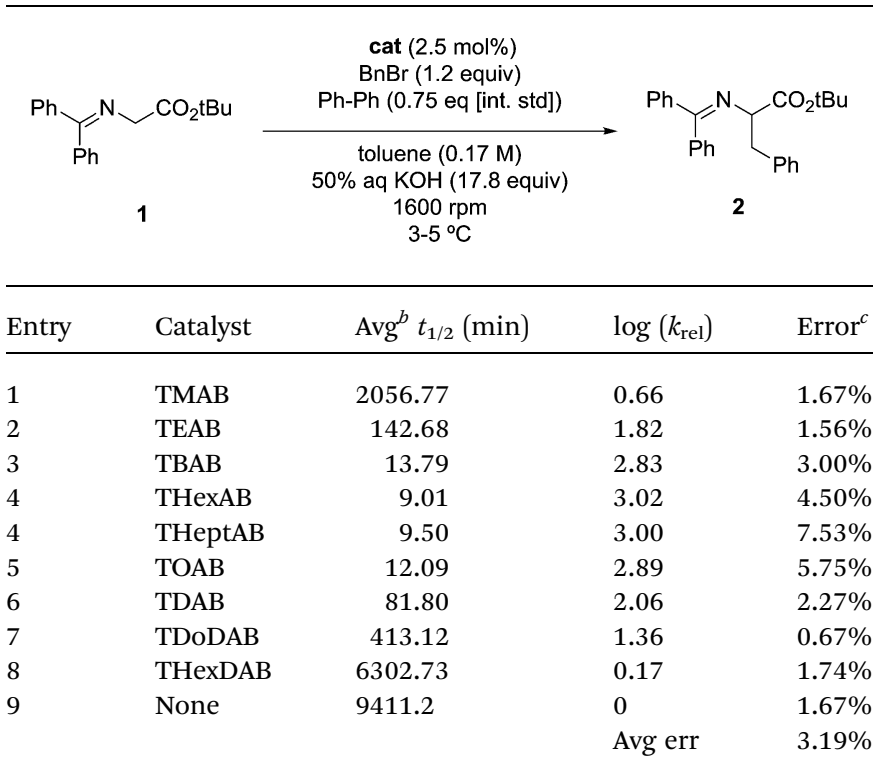

${ }^{a}$ All reactions performed on a $0.34 \mathrm{mmol}$ scale. ${ }^{b}$ Average of at least three runs. ${ }^{c}$ Error calculated as $\operatorname{stddev}\left(\log \left(t_{1 / 2}\right)\right) / \log \left(t_{1 / 2}\right)_{\text {avg }}$ and reported as percentage.

change of rate with respect to XSA being non-symmetrical between the low XSA and high XSA catalyst regimes.

The correlation between ammonium ion accessibility and rate was investigated using the solvent accessible ammonium ion surface area (NC4_SA) and $\boldsymbol{q}$ descriptors. Plotting the rate data with respect to the solvent accessible charged surface area (NC4_SA) showed a moderate bilinear correlation with an $R^{2}$ of 0.886 (Fig. 8a). The lack of NC4_SA variation for the longer-chain $\mathrm{R}_{4} \mathrm{~N}^{+}$catalysts results in an insufficient model. Interestingly, a bilinear relationship between $\boldsymbol{q}$ and rate does exist, with a high degree of correlation ( $R^{2}$ of 0.961 ) (Fig. 8b). The fit is not as precise as that observed between XSA and $\log \left(k_{\mathrm{rel}}\right)$, but the correlation exists nonetheless.

The existence of a highly correlated bilinear relationship between $\boldsymbol{q}$ and rate is perplexing in view of the relatively poor correlation observed between NC4_SA and rate. The NC4_SA descriptor is an actual measure of ammonium ion accessibility and if $\boldsymbol{q}$ measured ammonium ion accessibility, it would be expected to give a moderately correlated model as well. Thus, the superior correlation of $\boldsymbol{q}$ and rate indicates that the $\boldsymbol{q}$ descriptor, while operationally simple in calculation, encompasses a wider range of properties than ammonium ion accessibility in the traditional sense.

The existence of the relationships between rate, XSA, and $\boldsymbol{q}$ suggested a correlation between XSA and $\boldsymbol{q}$. Indeed, an excellent reciprocal model $\left(R^{2}=0.999\right)$ between XSA and $\boldsymbol{q}$ was found (Fig. 8c). This correlation indicates that for the straight-chain $\mathrm{R}_{4} \mathrm{~N}^{+}$catalysts, $\boldsymbol{q}$ is an approximation of the inverse of XSA. The reciprocity arises due to catalysts with large XSA values generally containing long alkyl chains, giving a smaller value of $\boldsymbol{q}^{21}$ This leads to the conclusion that $\boldsymbol{q}$ is not simply a measure of ammonium ion accessibility, but instead a measure of molecular behavior at an anisotropic interface between polar and 

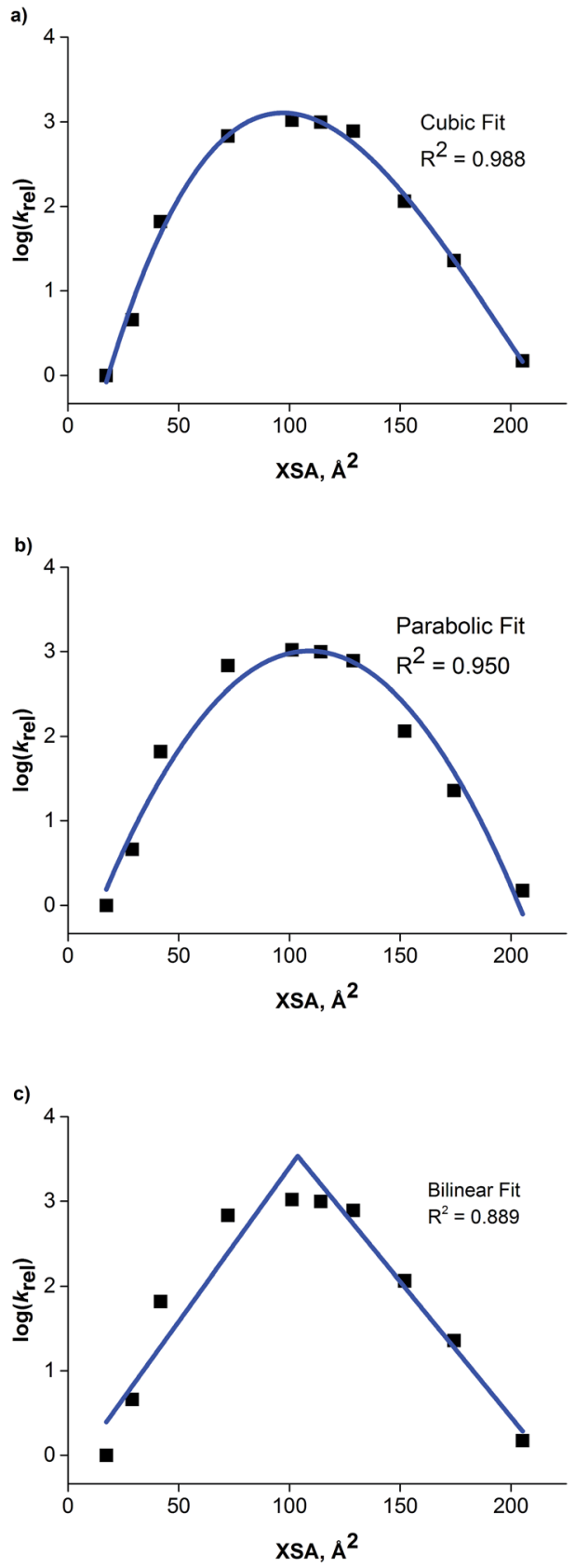

Fig. 7 (a) Cubic model between XSA and $\log \left(k_{\text {rel }}\right)$. (b) Parabolic model between XSA and $\log \left(k_{\text {rel }}\right)$. (c) Bilinear model between XSA and $\log \left(k_{\text {rel }}\right)$.

nonpolar solvation regions; a set of properties XSA was designed to represent as a descriptor.

The observed correlations suggest that XSA describes the behavior of $\mathrm{R}_{4} \mathrm{~N}^{+}$ions, specifically the ability to adsorb and desorb from the interface. Envisioning the adsorption/desorption process as an equilibrium between the ion pair adsorbed at the interface and the ion pair residing in the organic phase leads to two transport-rate constants, $k_{1}^{\prime}$ (desorption) and $k_{-1}^{\prime}$ (adsorption) (Fig. 9). The observed rate $\left(k_{\text {obs }}\right)$ arises from a combination of $k_{1}^{\prime}$ and $k_{-1}^{\prime}$ The model obtained suggests that $\log \left(k_{\text {obs }}\right)$ is correlated to XSA. The relationship between XSA and rate, which contains both positive and negative slopes, indicates

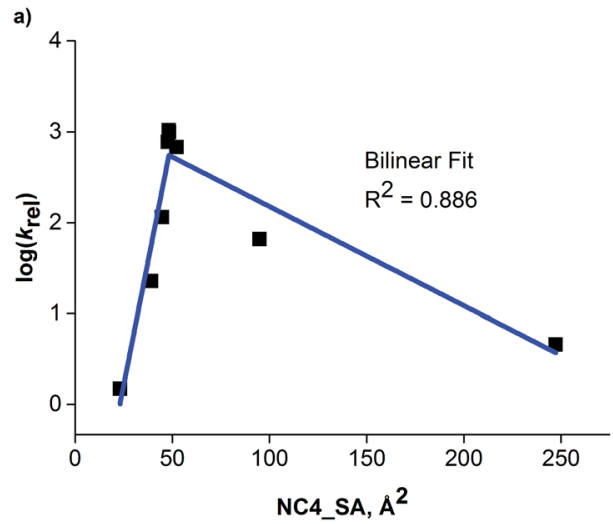

b)
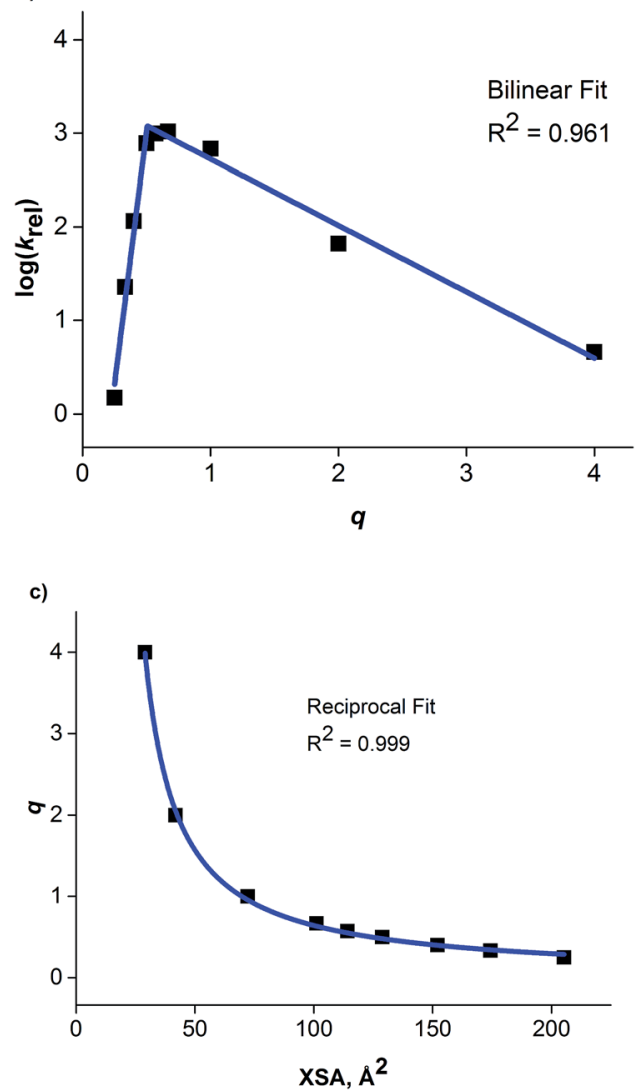

Fig. 8 (a) Plot of NC4_SA against observed rate. (b) Bilinear relationship observed between ammonium ion accessibility $(q)$ and observed rate. (c) Reciprocal relationship between XSA and $q$.

a change in the transport-rate limiting step in the mechanism of the reaction. It should be noted that the non-linearity of the rate changes and the subsequent cubic model selection does not change the physical interpretation that follows, as this is based on the change in positive to negative slopes as XSA increases, and not on the rate of change of the slope. ${ }^{22}$

In the positive slope regime (XSA $<75 \AA^{2}$ ), desorption from the interface ( $k_{1}^{\prime}$ in Fig. 9a) is the expected slow step as observed in the original study. As the smaller $\mathrm{R}_{4} \mathrm{~N}^{+}$catalysts are hydrophilic (and less soluble in organic solvents), the energy barrier to achieve desorption from the interface is larger than the analogous process for adsorption to the interface, and thus the rate of desorption is 


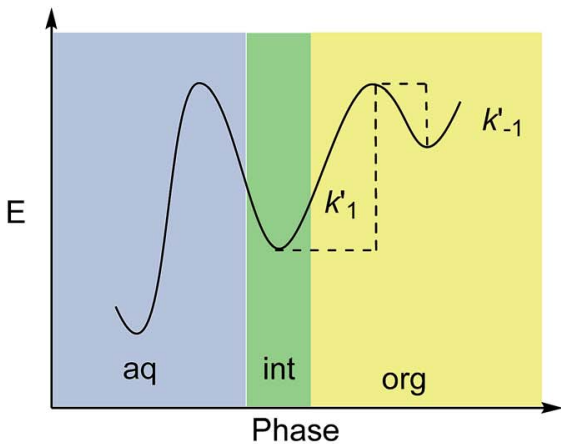

(a)

XSA $<75 \AA^{2}$

Observed

$\log \left(k_{o b s}\right)=f(X S A)$

Interpreted as

$\mathrm{f}(\mathrm{XSA}) \propto k_{1}^{\prime} k_{-1}^{\prime}$

$k_{-1}^{\prime}$ fast

$k_{1}^{\prime}$ increasing

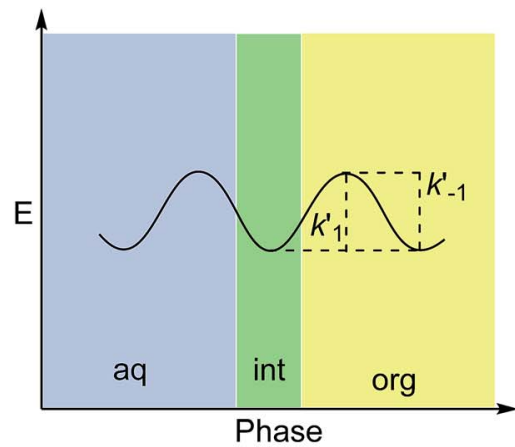

(b)

\section{$75<\mathrm{XSA}<140 \AA^{2}$}

Observed

$\log \left(k_{\text {obs }}\right)$ ind. of XSA

Interpreted as

$\mathrm{f}(\mathrm{XSA}) \propto k^{\prime} k_{-1}^{\prime}$

$k_{1}^{\prime}=k_{-1}^{\prime}$

diffusion limiting

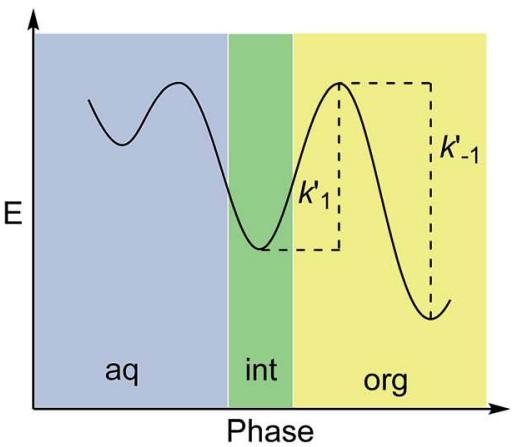

(c)

$$
\begin{gathered}
X S A>140 A^{2} \\
\text { Observed } \\
\log \left(k_{\text {obs }}\right)=f(X S A) \\
\underline{\text { Interpreted }} \underline{\text { as }} \\
\mathrm{f}(\mathrm{XSA}) \alpha k_{1}^{\prime} k_{-1}^{\prime} \\
k_{1}^{\prime} \text { fast } \\
k_{-1}^{\prime} \text { increasing }
\end{gathered}
$$

Fig. 9 Physical interpretation of XSA with relationship to transport-rate limiting PTC mechanism: (a) desorption is the transport-rate limiting step for small $\mathrm{R}_{4} \mathrm{~N}^{+}$salts. (b) Both desorption and adsorption processes are diffusion rate limited (fast). (c) Adsorption becomes the transport-rate limiting step as the $\mathrm{R}_{4} \mathrm{~N}^{+}$become larger. ${ }^{20}$ The function $f(\mathrm{XSA})$ is representative of the cubic model obtained between XSA and log $\left(k_{\text {rel }}\right)$. Note that the physical interpretation of positive and negative slope regimes is not dependent on the model used to predict the rate.

the transport-rate limiting step. In the negative slope regime (XSA $>140 \AA^{2}$ ), decreasing rate with increasing XSA suggests that adsorption to the interface $\left(k_{-1}^{\prime}\right.$ in Fig. $\left.9 \mathrm{c}\right)$ is the transport-rate limiting step. Since desorption should continue to be fast for the more lipophilic catalysts $\left(k_{1}^{\prime}\right.$ is large), slow adsorption of the catalyst to the interface must be responsible for the decrease in rate; the energy barrier to achieve adsorption is likely lower for the smaller, more hydrophilic catalysts. The similar rates of catalysts at the inflection region $\left(75<\mathrm{XSA}<140 \AA^{2}\right)$ indicates that the rate of adsorption and desorption of the ion pairs are both fast, and likely diffusion limited (Fig. 9b). The trends in rate with respect to catalyst suggest that $k_{\mathrm{obs}}$ is proportional to $k_{1}^{\prime} k_{-1}^{\prime}$.

Because of the design of the XSA parameter as an estimation of molecular behavior under highly anisotropic conditions at an interface between polar and nonpolar environments and the observed correlation between XSA and rate, it is proposed that XSA represents the affinity of the catalyst at the interface (adsorption to interface) as well as affinity of the catalyst-enolate $\left(\mathbf{Q}^{+} \mathbf{E n}^{-}\right)$ion pair for the organic phase (desorption from interface) (XSA correlates with $\left.k_{1}^{\prime} k_{-1}^{\prime}\right){ }^{23}$ The proper balance of these factors leads to high catalytic activity, which is observed in the $75<$ XSA $<$ $140 \AA^{2}$ region; the highest rates are observed with catalysts of intermediate XSA, which likely exhibit diffusion rate limited transfer to and from the interface. Moreover, because of the correlation between $\boldsymbol{q}$ and XSA, it can be proposed that $\boldsymbol{q}$ is not a simple measure of the accessibility of the ammonium ion, but rather a reflection of the behavior of the ions at the interface. As shown, XSA appears to measure these same fundamental properties, but with better correlation with rate. Most importantly the XSA model can be applied to all types of ammonium ions. This feature means that XSA may be used to select and predict highly active catalysts. The previous report showed that XSA displayed the same correlation for rate with several cyclopentapyrrolizidine and cinchona type PTC catalysts. ${ }^{11}$ Unfortunately, the lack of larger XSA catalysts in these subclasses precludes the ability to explore further correlation in the high XSA regime.

\section{Conclusions}

The correlation observed between XSA and $\log \left(k_{\text {rel }}\right)$ serves to validate the prediction made in the initial report from these laboratories. By investigating quaternary ammonium salts with larger cross-sectional areas, a cubic (non-linear) relationship has been found between XSA and rate, indicating that XSA is sufficient as a single descriptor to predict transport-limiting PTC rates. Furthermore, the observed relationship with rate indicates that XSA captures the complicated behavior of the $\mathbf{Q}^{+}$ species at the interface in transport-limited PTC reactions of enolates. The observed relationship between $\boldsymbol{q}$ and both rate and XSA indicates that $\boldsymbol{q}$ models the same interfacial behavior instead of the physical accessibility of the ammonium ion. The observed correlations and derived models lead to the conclusion that XSA is an excellent surrogate for $\boldsymbol{q}$ in more complicated quaternary ammonium ion species. Further investigation will be required to show the utility of XSA to predict rate in other transport-limiting PTC reactions.

\section{Acknowledgements}

We are grateful to the W. M. Keck Foundation for generous financial support. 


\section{Notes and references}

1 (a) P. Anastas and J. Warner, Green Chemistry: Theory and Practice, Oxford University Press, Oxford, UK, 1998; (b) W. P. Weber and G. W. Gokel, Phase Transfer Catalysis in Organic Synthesis, Springer-Verlag, Berlin, New York, 1977, vol. 4; (c) E. V. Dehmlow and S. S. Dehmlow, Phase Transfer Catalysis, Verlag Chemie, Weinheim, Deerfield Beach, 1983, vol. 11; (d) Phase-Transfer Catalysis: New Chemistry, Catalysts, and Applications, ed. C. M. Starks, American Chemical Society, Washington, DC, 1985; (e) C. M. Starks, C. L. Liotta, and M. Halpern, Phase-Transfer Catalysis: Fundamentals, Applications and Industrial Perspectives, Chapman and Hall, New York, 1994; (f) Phase-Transfer Catalysis: Mehcanism and Syntheses, ed. M. E. Halpern, American Chemical Society, Washington, DC, 1997; $(g)$ M. Makosza and M. Fedorynski, in Interfacial Catalysis, ed. A. G. Volkov, Marcel Dekker, New York, 2003, pp. 150-201.

2 Handbook of Phase Transfer Catalysis, ed. Y. Sasson and R. Neumann, Chapman and Hall, London, 1997.

3 For an insightful analysis of hydroxide-initiated PTC, see ref. 1e, pp. 89-108.

4 (a) D. L. Hughes, in Comprehensive Asymmetric Catalysis, ed. E. N. Jacobsen, A. Pfaltz and H. Yamamoto, Springer Verlag, Heidelberg, 1999, vol. III, ch. 34.1; (b) D. L. Hughes, in Comprehensive Asymmetric Catalysis, ed. E. N. Jacobsen, A. Pfaltz and H. Yamamoto, Springer Verlag, Heidelberg, 2004, supp. 1, pp. 161-170; (c) G. Helmchen, in Asymmetric Synthesis-The Essentials, ed. M. Christmann and S. Bräse, Wiley-VCH, Weinheim, 2006, pp. 95-99.

5 C. M. Starks and R. M. Owens, J. Am. Chem. Soc., 1973, 95, 3613-3617.

6 (a) M. Makosza, Tetrahedron Lett., 1966, 38, 4621-4624; (b) M. Makosza, Pure Appl. Chem., 1975, 43, 439-462; (c) M. Makosza and E. Bialecka, Tetrahedron Lett., 1977, 18, 183-186.

7 For the most recent summary of the state of mechanistic understanding of phase transfer catalysis, see: M. Makosza and M. Fedorynski, Catal. Rev., 2003, 45, 321-367.

8 See ref. $1 e$, pages 266-338.

9 (a) M. Halpern, Y. Sasson, I. Willner and M. Rabinovitz, Tetrahedron Lett., 1981, 22, 1719-1722; (b) M. Halpern, Y. Sasson and M. Rabinovitz, J. Org. Chem., 1983, 48, 10221025; (c) M. Halpern, Y. Sasson and M. Rabinovitz, J. Org. Chem., 1984, 49, 2011-2012; (d) M. Halpern, D. Feldman, Y. Sasson and M. Rabinovitz, Angew. Chem., 1984, 96, 7980; (e) M. Halpern, H. A. Zahalka, Y. Sasson and M. Rabinovitz, J. Org. Chem., 1985, 50, 5088-5092; $(f)$ D. Feldman, M. Halpern and M. Rabinovitz, J. Org. Chem., 1985, 50, 1746-1749.

10 M. Halpern, in Phase-Transfer Catalysis, Mechanism and Synthesis, ACS Symposium Series 659, ed. M. E. Halpern, ACS, Washington, DC, 1996, ch. 8.

11 (a) S. E. Denmark, N. D. Gould and L. M. Wolf, J. Org. Chem., 2011, 76, 4260-4336; (b) S. E. Denmark, N. D. Gould and L. M. Wolf, J. Org. Chem., 2011, 76, 4337-4357; (c) For details on the determination of stir rate dependence and ensuring a transport-limiting PTC regime, see ref. 11a; (d) C. M. Starks and R. M. Owens, J. Am. Chem. Soc., 1973, 95, 3613-3617; (e) C. M. Starks, J. Am. Chem. Soc., 1971, 93, 195-199.

12 The NC4_SA descriptors were coded in scientific vector language (SVL) utilizing a SMILES string search $([\mathrm{N}+](\mathrm{C})(\mathrm{C})(\mathrm{C}))$ and the AtomSurfaceArea function with variable probe radii. See $\mathrm{ESI} \dagger$ for details.

13 (a) M. J. O'Donnell, W. D. Bennett and S. D. Wu, J. Am. Chem. Soc., 1989, 111, 2353-2355; (b) M. J. O'Donnell, W. D. Bennett, W. A. Bruder, W. N. Jacobsen, K. Knuth, B. LeClef, R. L. Polt, F. G. Bordwell, S. R. Mrozack and T. A. Cripe, J. Am. Chem. Soc., 1988, 110, 8520-8525; (c) M. J. O'Donnell, Acc. Chem. Res., 2004, 37, 506-517.

14 G. Gerebtzoff and A. Seelig, J. Chem. Inf. Model., 2006, 46, 2638-2650.

15 (a) H. Fischer, R. Gottschlich and A. Seelig, J. Membr. Biol., 1998, 165, 201-211; (b) A. Seelig, J. Mol. Neurosci., 2007, 33, 32-41; (c) A. Seelig, R. Gottschlich and R. M. Devant, Proc. Natl. Acad. Sci. U. S. A., 1994, 91, 68-72; (d) M. J. Rosen, Surfactants and Interfacial Phenomena, John Wiley \& Sons, New York, 1989.

16 K. Maruoka, in Asymmetric Phase Transfer Catalysis, ed. K. Maruoka, Wiley-VCH, Weinheim, 2008, ch. 5.

17 For both NC4_SA and XSA 3D descriptors, the given values are averages for the descriptor values of all conformers generated in a $20 \mathrm{kcal} \mathrm{mol}^{-1}$ window using MMFF94x. See $\mathrm{ESI} \dagger$ for details.

18 The error is calculated as $\operatorname{stddev}\left(\log \left(t_{1 / 2}\right)\right) / \operatorname{avg}\left(\log \left(t_{1 / 2}\right)\right) \times$ 100.

19 (a) The XSA, NC4_SA and $\boldsymbol{q}$ models were generated using statistical fitting methods in OriginPro 9. The cubic model was chosen as the best model using both the Akaike information criterion (AIC) and Bayesian information criterion (BIC) methods of model quality assessment. These statistical measures predict the relative quality of a series of models, balancing the simplicity of the model (terms in the equation) with the goodness of fit $\left(R^{2}\right)$. The higher the calculated value of AIC and BIC, the lower the quality of the model in a set. The set of models in this study are as follows: linear, bilinear, parabolic, cubic, and 4th order. Both AIC and BIC support cubic as the best model, with AIC suggesting the cubic model is $35 \times$ more likely to be the correct model over parabolic, and $1712 \times$ more likely to be a more correct model over a 4 th order polynomial. For more details, see ESI.†; $(b)$ For a general overview of AIC and BIC in statistical model selection, see: K. Aho, D. Derryberry and T. Peterson, Ecology, 2014, 95, 631-636

20 It should be noted that the interpretation of correlation between XSA and $k_{1}^{\prime} k_{-1}^{\prime}$ rests on the assumptions that the other rate constants in $k_{\text {obs }}$ lead to the log relationship.

21 The observed reciprocal relationship also suggests that XSA is proportional to chain length in the symmetrical $\mathrm{R}_{4} \mathrm{~N}^{+}$ salts. Indeed, a linear relationship is found between XSA and $\mathrm{C}_{\mathrm{n}}$. See ESI† for details. 
22 The unsymmetrical nature of the positive and negative slope regimes is primarily responsible for requiring a higher order polynomial. As this is a complicated system, it is likely that the change in adsorption/desorption ability do not change concurrently. See note ref. 19 for details on model selection and validation.
23 This correlation could be stated as a proportionality: $f(\mathrm{XSA}) \alpha$ $k_{1}^{\prime} k_{-1}^{\prime}$ where $f(\mathrm{XSA})$ is representative of the cubic function that describes the relationship between the XSA descriptor and rate. 